\title{
Michel LALLEMENT, L'Âge du faire. Hacking, travail, anarchie
}

Marc-Antoine Sabaté

\section{(2) OpenEdition}

\section{Journals}

Édition électronique

URL : https://journals.openedition.org/ress/4135

DOI : $10.4000 /$ ress. 4135

ISSN : 1663-4446

Éditeur

Librairie Droz

\section{Édition imprimée}

Date de publication : 14 décembre 2018

Pagination : 282-286

ISSN : 0048-8046

\section{Référence électronique}

Marc-Antoine Sabaté, « Michel LALLEMENT, L'Âge du faire. Hacking, travail, anarchie », Revue européenne des sciences sociales [En ligne], 56-2 | 2018, mis en ligne le 14 décembre 2018, consulté le 07 janvier 2022. URL : http://journals.openedition.org/ress/4135; DOI : https://doi.org/10.4000/ress.4135

Ce document a été généré automatiquement le 7 janvier 2022.

(c) Librairie Droz 


\title{
Michel LALLEMENT, L'Âge du faire. Hacking, travail, anarchie
}

\author{
Marc-Antoine Sabaté
}

\section{RÉFÉRENCE}

Michel LALLEMENT, 2015, L’Âge du faire. Hacking, travail, anarchie, Paris, Éditions du Seuil, « La couleur des idées », 442 p.

1 Le « bidouillage », le « hack », ce « travail pour soi » pratiqué dans les Hackerspaces, Tech Shops et autres Fab Labs, porte-t-il en germe une reconfiguration de notre rapport collectif au travail? Telle est la question qui motive l'ouvrage riche et stimulant que Michel Lallement, s'appuyant sur une étude menée au sein de Noisebridge, hackerspace de la baie de San Fransisco, consacre à « l'âge du faire » dans lequel nous serions entré.

Entre programmation informatique et impression 3D, construction de robots et détournement à portée artistique, éducative ou politique d'objets en tous genres, il s'agit pour le sociologue de délimiter un " paradigme du faire » aux contours fuyants, de le mettre à l'épreuve, en observant non seulement « ce que font les individus et les groupes », mais aussi «les représentations sur lesquelles ils s'appuient pour cela » (p. 216). C'est donc toute une "morale de l'action", celle de l'éthique hacker, qui se trouve placée au cœur de l'enquête. Une éthique dont on pourrait dire qu'elle repose sur trois piliers : la «volonté de créer et de partager en se défaisant des contraintes imposées par le marché »; la quête " des moyens et des espaces d'activité où le travail est à lui-même sa propre fin » (p.12); la conviction partagée que, "parce qu'ils promettent un autre rapport à soi et aux autres, les objets et les bidouillages dont ils sont les supports sont d'authentiques vecteurs d'émancipation individuelle et collective » (p. 125).

Divisé en trois temps (histoire, enquête, critique), le travail de Lallement chemine en réalité à travers deux grandes questions: qu'est-ce qu'un hacker? en quoi les hackerspaces bouleversent-ils la division et l'organisation du travail ? 
Premier enjeu donc: de quoi le hack est-il le nom? Problématique transversale à l'ensemble du livre, elle se résume à deux controverses structurantes: "le mode d'activité à privilégier » et "le matériau à hacker en priorité »(p.338-339). Tout d'abord, et contrairement à l'usage courant du terme, l'immense majorité de ceux qui se revendiquent hackers prennent explicitement leurs distances avec les crackers, ces pirates «qui agissent dans l'illégalité la plus complète pour pénétrer des réseaux privés, piller des informations ou mettre à sac des systèmes informatiques » (p. 13). Ces derniers "détruisent» ce que les hackers "construisent» (p. 347-348). Il existe pourtant une réelle porosité entre ces deux mondes, certaines pratiques pirates rejoignant l'éthique hacker dans la lutte contre le pouvoir bureaucratique et pour la libre circulation des informations.

5 Au-delà de ce partage, "une interrogation récurrente » fait l'objet d'une discussion sans fin: «qui est hacker et qui ne l'est pas?» (p. 250) Le hack est tout d'abord identifiable à ce qu'il est à la fois un « défi » et un " plaisir (fun)». L'efficacité constitue par ailleurs un critère essentiel : « Un hack est d'abord un geste simple mais aux effets majeurs ». On ne saurait pourtant voir dans ce «faire vite et bien» un impératif de "productivité » ou "encore moins de rentabilité». Il tient plutôt à une quête d'élégance formelle, spécialement chez les programmeurs, où ce qui importe pardessus tout est la simplicité «de la solution utilisée pour résoudre un problème complexe ». On touche ici à la dimension esthétique de l'activité : « hacker, c'est refuser de dissocier le travail et l'art ». La pratique du hacking demande dès lors l'acquisition de certaines compétences, et il faut donc "faire ses preuves" pour obtenir la reconnaissance d'un monde qui se fonde ouvertement "sur un principe de méritocratie » (p. 217-229).

Reste encore à déterminer la destination du hack ainsi défini. S'il a toujours concerné le hardware autant que le software, la fabrication de circuits imprimés ou de microordinateurs autant que le codage, l'informatique en a néanmoins longtemps constitué le support privilégié. Épousant la mouvance makers et l'émergence du do it yourself, le bidouillage se tourne pourtant de manière croissante vers la fabrication/impression d'objets et de machines diverses (p. 49-54). Cet élargissement renouvelle également la déclinaison militante du faire. Une " tension structurelle » oppose dès lors «les «purs " informaticiens» aux «militants politiques». Pour les premiers, le hacking, s'il est en effet un levier d'émancipation, est essentiellement vécu comme "une forme de spiritualité » et « un art de vivre » à l'élitisme assumé (p. 357). Pour les seconds, il s'agit au contraire d'une activité fondamentalement politique, d'une réappropriation de soi et du monde qui, si elle passe bien-sûr par la bidouille technologique, ne saurait être circonscrite à un domaine particulier. À travers le hack de programmes, d'objets, mais aussi de vêtements ou de nourriture, c'est alors la société elle-même qu'il s'agit de transformer.

7 Pour le comprendre, il est nécessaire de s'intéresser à la seconde question qui traverse l'ouvrage, celle de l'organisation du faire au sein des hackerspaces. Le hacking ne saurait en effet se réduire à un contenu. Toute activité de travail est inséparable du lieu où elle est mise en œuvre et où elle s'organise, donnant ainsi forme à ce contenu. Pas de hacking sans hackerspaces (voir à ce propos la présentation filmée du hackerspace réalisée par Noisebridge et disponible sur Youtube : <https://www.youtube.com/watch? $\mathrm{v}=2 \mathrm{wGMjRJpLCA}>$ ). 
Un «sentiment d'appartenance communautaire » s'y développe, mais sur un mode paradoxal, sans «interconnaissance approfondie» (p.193). De fait, mis à part les moments "rituels" où la communauté se regroupe (réunions hebdomadaires, conférences, etc.), « les relations de face à face sont en général peu intenses. » (p. 208) Les échanges sont au contraire très fournis en ligne. Alone together, "seuls en groupe »: hacker est une "pratique solitaire dans bien des cas, mais toujours accomplie dans un environnement collectif» (p. 178). La simple "co-présence" permet ainsi une " coopération adhocratique ", " basée sur de l'implication fluide et volontaire » : chacun rejoignant ou se retirant spontanément d'un projet selon son envie (p. 248-249). Offrant à ses membres des ressources matérielles et symboliques pour mener à bien leurs projets, le hackerspace est donc bien un "vecteur d'intégration sociale », et donne lieu à une "forme d'individualisme positif", une certaine manière "d'être libres ensemble » (p. 210-213).

Communauté de projet, la vie du hackerspace n'en est pas moins structurée par des règles. Leur application donne toutefois lieu à des tensions récurrentes, dont la résolution occupe une part considérable du temps collectif. Formellement, une seule «règle de fonctionnement » est censée régir les interactions: "Be excellent to each other ». Qu'en est-il en pratique? Deux éléments méritent d'être mentionnés. Le premier est l'usage anarchiste du consensus. L'étude décrit avec subtilité tous les avantages et les inconvénients de la "décision consensuelle» pour la communauté. Retenons simplement la manière dont elle entre en conflit avec un second principe d'action. Il s'agit de ce que les hackers nomment «do-ocratie » : le pouvoir à ceux qui font (p. 268). Comment concilier cette valorisation de l'initiative individuelle avec la pratique du consensus? En réalité, des «hiérarchies» plus ou moins formelles (rôle prééminent des " anciens ») finissent par forcer la décision, et structurent le monde hacker sur un autre mode que celui du simple mérite do-ocratique (p. 282).

Pour Lallement, cet «inévitable décalage entre l'idéal régulateur et les pratiques sociales " n'empêche pas de conclure que «les hackers ont réussi à donner vie à un modèle de pratiques alternatives.» (p. 251) Deux éléments permettent finalement de l'appréhender.

11 Un premier constat s'impose: "tout comme l'éthique protestante d'hier a pu entretenir une affinité élective avec l'esprit du capitalisme industriel, celle des hackers d'aujourd'hui peut aisément entrer en correspondance avec les valeurs que portent nombre d'entrepreneurs de la Silicon Valley.» (p.314-315) Il ne s'agirait au fond que des deux faces de la même pièce. Le tandem des fondateurs d'Apple, Steve Wozniak et Steve Jobs, en fournit la plus éclatante illustration : le premier incarnant par excellence le génie du bidouilleur esthète ; le second celui du capitaliste stratège (p. 134-135). Pour l'auteur, cependant, «l'engouement pour le faire n'est pas que la simple expression d'une nouvelle ruse de la raison capitaliste » (p. 21). De fait, le rapport que les hackers entretiennent avec le marché «demeure principalement instrumental» (p. 399), comme en témoigne la typologie d'inspiration weberienne construite par l'auteur pour dégager les différents profils de hackers (p. 309-325). Lallement va même jusqu'à suggérer une inversion de dynamique : lorsque les années 1980-1990 auraient en effet vu quelque chose comme un détournement de la critique artiste (voir Luc Boltanski et Ève Chiapello, 1999, Le Nouvel Esprit du capitalisme, Paris, Gallimard) par un capitalisme «capable d'exploiter la subjectivité et la créativité », tout indiquerait que «les hackers 
d'aujourd'hui pratiquent à leur tour l'art du judo social en se servant du marché comme d'un soutien au profit de l'innovation contestataire » (p. 400).

Cette transformation subversive apparaît plus claire encore à l'examen d'un deuxième enjeu : la division du temps social sur laquelle repose le faire. L'auteur s'appuie ici sur les réflexions menées par André Gorz à partir des années 1980 (voir notamment Adieux au prolétariat. Au-delà du socialisme, Paris, Galilée, 1980). À première vue, les hackers semblent offrir un exemple sans pareil du dualisme autonomie/hétéronomie défendu par ce dernier. Pour la plupart, en effet, l'effectuation d'un travail socialement nécessaire dans la sphère de l'hétéronomie est subordonnée aux objectifs de la sphère autonome ; elle sert matériellement d'appui à la réalisation de projets personnels, nonnécessaires et laissant libre cours à l'imagination de chacun. Lallement s'inscrit pourtant finalement en faux vis-à-vis de ce "fil gorzien ", suivant lequel «seules les activités échappant à l'emprise systémique sont dignes d'être considérées comme autonomes", et qui conduirait ainsi à négliger, "par principe ", les "capacités dont savent faire preuve les individus et les groupes pour instrumentaliser les systèmes sociaux à leurs propres profits ». Il ne s'agirait dès lors pas seulement de penser "l'autonomie du travail ", mais bien «l'autonomie au travail», «l'autonomie dans l'hétéronomie » (p. 403-405).

L'autonomie n'est en effet pas que la « capacité à produire ses propres règles ». Elle est tout autant capacité «à user de règles hétéronomes en toute liberté", ce dont témoignerait avec éloquence la pratique hacker, démontrant ainsi « qu'il est possible de donner vie au travail pour soi tout en se frottant activement au marché et à ses contraintes multiples. » (p. 409) De fait, par un mimétisme ludique et subversif qui leur est propre, allant du «nom du hackerspace » aux «moyens de communication » et de partage des connaissances, en passant par les "codes vestimentaires», les hackers trouvent dans « le détournement interne des règles édictées » par les mondes en marge desquels ils se situent - l'entreprise et l'université -, « un ensemble de ressources symboliques » qui leur permettent d'exister en tant que collectifs de travail autonomes.

Le livre débouche finalement sur l'ébauche d'un "programme de recherche ", celui d'une « sociologie des utopies réelles». On s'interrogera, pour conclure, sur la tension inexplorée à laquelle fait face un travail qui, placé sous le haut patronage d'Émile Durkheim (comme en témoigne l'exergue tirée des Formes élémentaires de la vie religieuse), inscrit son analyse des "poches alternatives", des contre-lieux "où déjà fermente le nouveau monde ", dans la continuité de la science des "hétérotopies » souhaitée par Michel Foucault. Ne faudrait-il pas, en effet, approfondir l'écart entre deux manières de penser la normativité immanente aux pratiques sociales - une sociologie se rapportant à un social substantivé face à une pensée critique focalisée sur les jaillissements singuliers de la praxis -, pour mesurer ce que le faire fait à la division du travail? 


\section{AUTEURS}

\section{MARC-ANTOINE SABATÉ}

Université libre de Bruxelles, Centre de Théorie Politique 